$\frac{12}{3-18}-96850$

UCRL-ID-123071

\title{
RECEIVED
}

\author{
MAR 181996 \\ OSTI
}

\section{A Survey of Models for the Prediction of Ambient Ocean Noise: Circa 1995}

\author{
R. Doolittle, Consultant \\ Imaging and Detection Program
}

January 1996

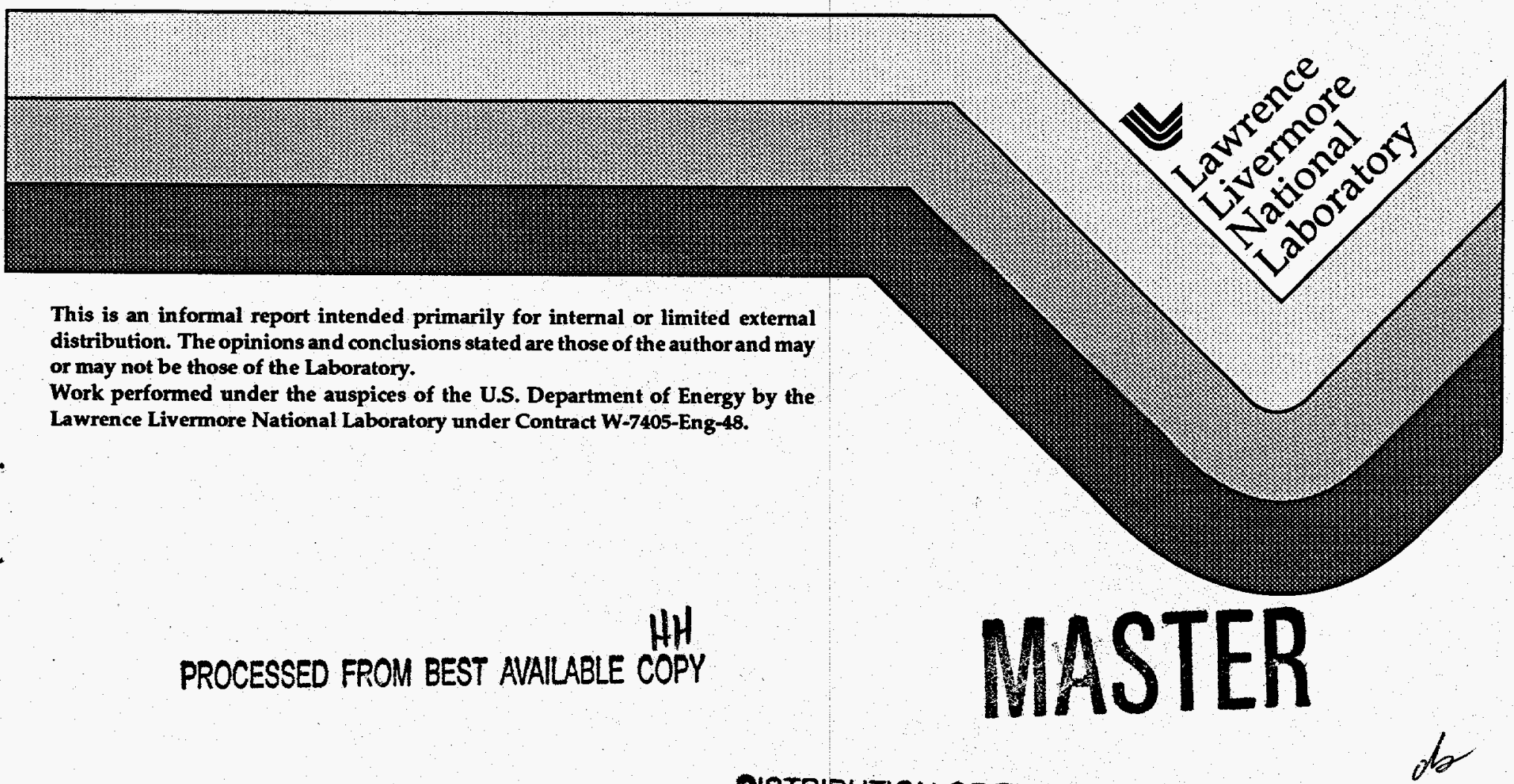




\section{DISCLAIMER}

This document was prepared as an account of work sponsored by an agency of the United States Government. Neither the United States Government nor the University of California nor any of their employees, makes any warranty, express or implied, or assumes any legal liability or responsibility for the accuracy, completeness, or usefulness of any information, apparatus, product, or process disclosed, or represents that its use would not infringe privately owned rights. Reference herein to any specific commercial product, process, or service by trade name, trademark, manufacturer, or otherwise, does not necessarily constitute or imply its endorsement, recommendation, or favoring by the United States Government or the University of California. The views and opinions of authors expressed herein do not necessarily state or reflect those of the United States Government or the University of California, and shall not be used for advertising or product endorsement purposes.

This report has been reproduced directly from the best available copy.

Available to DOE and DOE contractors from the Office of Scientific and Technical Information P.O. Box 62, Oak Ridge, TN 37831

Prices available from (615) 576-8401, FTS 626-8401

Avalable to the public from the National Technical Information Service

U.S. Department of Commerce 5285 Port Royal Rd., Springfield, VA 22161 


\section{DISCLAMMER}

Portions of this document may be illegible in electronic image products. Images are produced from the best available original document. 
A Survey of Models for the Prediction of Ambient Ocean Noise:

Circa 1995

1. Introduction

2. Shallow Water Model Building \& Validation
A. Physical Models
B. Stochastic Models

3. Recent Models

A. Cron and Sherman

B. Kuperman and Ingenito

C. Buckingham

D. JKPS treatment

E. Carey, et. al.

F. Piggott

4. Measured Noise

5. Practical Models

6. Findings

7. Recommendations 


\section{INTRODUCTION}

The state of the art of model development for application to computer studies of undersea search systems utilizing acoustics is surveyed in this document. Due to the demands for surveillance of submarines operating in ocean basins, the development of noise models for application in deep oceans is fairly advanced and somewhat generic. This is due to the deep sound channel, discovered during World War II , which when present allows for long-range sound propagation with little or no interaction with the bottom. Exceptions to this channel, also well understood, are found in both the high latitudes where the sound is upward refracting and in tropical ocean areas with downward refracting sound transmission. The controlling parameter is the sound speed as a function of depth within the ocean, the sound speed profile. When independent of range, this profile may be converted to a noise-versus-depth profile ${ }^{1}$ with well-validated consequences for deepocean ambient noise.

When considering ocean areas of shallow water, the littoral regions, the idea of a generic ocean channel advisedly is abandoned. The locally unique nature of both the noise production mechanisms and of the channel carrying the sound, obviates the generic treatment. Nevertheless, idealizations of this case exist and promote the understanding if not the exact predictability of the statistics of shallow water ambient noise. Some examples of these models are given in this document.

As far as current US Navy interest is concerned, there appears to be no accepted standard model for ambient noise in shallow water. There are several candidates for this title, identified by acronyms derived from their deep-water counterparts, such as ANDES (Ambient Noise Directionality Estimation) and RANDII (Random Ambient Noise Directionality....). This class of models utilize estimates of the distribution of noise sources, coupled with propagation models, based primarily on normal modes, and accommodates directional receivers as measuring devices. Reflecting past priorities, these models are 
narrowband in temporal frequency. Broadband versions of this class of model are under development. $^{2}$

Interest in noise modeling is at $\mathrm{ebb}$ at the Office of Naval Research, the principal Navy sponsor ${ }^{3}$ of such programs. ${ }^{4}$ There is more interest in the area of noise modeling at the Naval Research Laboratory ${ }^{5}$ where a noise measurement program is underway but with no particular development work in noise modeling. Given the importance of sound propagation to noise modeling it is not surprising to find much support for work in sound propagation in shallow water at ONR.

There have been measurements at Duck Pier in North Carolina, using a bottomed eightelement acoustic receiving array cut for $600 \mathrm{~Hz}$ center frequency. Measurements were made of the noise under varying wind and surf conditions. Noise volumes (noise bearing, depth and frequency) were generated indicating a strong arrival of sound energy from the beach when surf was running and lesser energy during calm sea periods. Swell from an approaching hurricane also was observed. Storm conditions eventually caused the measurements to end. ${ }^{6}$

In discussions with Dick Heitmeyer he identified two types of noise models which he terms as physics-based and stochastic. We make use of this dichotomy although it is recognized that physical arguments, are based in part on stochastic reasoning.

Physics-based Approach.

The physics-based approach attempts to identify sources of noise and to characterize these sources in terms of their physical extent, source strength, frequency spectrum and directionality. This modeling proceeds by developing the underlying theoretical physics and identifying parameters that account for all of the significant effects with their range of variation. Usually, a computer simulation is produced by implementation of the theoretical formulas as the end product of such a theory. The theory is tested for validity by comparing this simulation to data from existing or devised experiments. 
All sources of noise that are believed to be significant are included in this approach which usually assumes mutual independence between all sources so that, in any implementation of source fields, the resulting noise effects are additive. Noise sources include wind and waves, shipping and other industrial activity such as fishing, oil platform operations, harbor traffic and port activity, recreational boating and biologics.

The physics-based approach requires that several key aspects be addressed properly. These aspects include: characterization of sources of sound; distribution of the sources in spacetime; response of an acoustic channel with boundaries to a source within or on the boundary of the undersea channel. Both space-time continuous and discrete source types must be considered. The eventual aim is to produce a computed time series of acoustic potential (or more commonly, pressure) at a receiver or array of receivers which passes available tests for realism and allows predictions of the performance measures of arrays such as noise gain of a receiving hydrophone array.

The physics-based approach finds its most direct application in search system studies where, initially, an undersea environment and a search scenario are defined with parameter values chosen for a given geographical region. The state of the art models for undersea ambient noise using this approach are the before-mentioned ANDES-SAIC and RANDII-Saclant Center.

Stochastic Approach

The stochastic approach attempts to develop an understanding of the noise probability distribution in space-time. While this may be defined formally, it is impractical to solve for the whole distribution and simplifications are sought using restricting conditions and assumptions.

One alternative is to look for a probability distribution with certain properties which follow from physical arguments or some other rational basis. For instance, in the case of 
Brownian motion, the agitation agent is thermal so that for small particles we might expect the particle kinetic energy to have thermal fluctuations.

\begin{abstract}
A simple example ${ }^{7}$ is one in which the assumptions lead to the Gaussian distribution. We seek the distribution with maximum Shannon entropy and a fixed mean square. The mean square value of a single variate is fixed (Woodard suggests that this may correspond to fixing the mean noise voltage or pressure.) Then for a distribution $p$ (with the assessory integrals, $\int p d x=1$ and $\int x^{2} p d x=\sigma^{2}$ ) the Shannon entropy, $-\int p \log p d x$, is maximized by the method of Lagrange multipliers. The solution to this maximization problem is $N[0, \sigma]$. On the basis of Shannon entropy as a measure of randomness, the gaussian distribution with fixed mean square is the most random of all. ${ }^{8}$ By identifying mean square with variance (for zero mean noise) and noise variance with noise mean power, we may state that the noise distribution with fixed mean power is the most random of all.
\end{abstract}

Many other assumptions and restrictions lead to other noise distributions. as is discussed by Middleton and others, Table 1 lists some of these from Woodard.

\title{
Table 1. Some Distributions and their Shannon Entropy
}

$\begin{array}{lll}\text { Assesory Conditions } & \text { Distribution } & \text { Shannon Entropy } \\ x \in[-X / 2, X / 2] & \text { Uniform } & \log X \\ \text { Fixed mean, } x<0 & \text { Exponential } & 1-\log (1 / 2) \\ \text { Fixed Mean Square } & \text { Normal, } N[0, \sigma] & \log \left(\sigma(2 \pi \mathrm{e})^{1 / 2}\right)\end{array}$

Other nongaussian noise distributions were developed and discussed by Middleton ${ }^{9}$

The most cogent application for the results of the stochastic approach is in system theory and practice where an input noise distribution is given and the output distribution is sought for a specified system response transformation. Transformation of noise processes, gaussian and nongaussian, has a rich literature. 
Although no special effort is being made to extend existing deep-water noise distribution models to shallow water it would seem that the effects of background noise must be included for effective studies of search systems in shallow water. This specially true whenever those systems, whether passive or active are noise limited ${ }^{10}$. There is a need for shallow water noise distribution theory and simulation and such theory stands apart from its deep-water form.

One reason for this need is that noise, even additive noise, alters the distribution of a signal and thus, as I. Dyer writes," "the distribution of signal plus noise is neither that of the signal alone nor that of the noise alone."

Another reason is that noise can be inverted to estimate regional geophysical properties of the subottom in shallow water. ${ }^{12}$ For these and other reasons the study of noise distributions and the building of noise models is of central importance in the treatment of underwater signal processing.

Furthermore, it is clear that ocean ambient noise in shallow water is non-gaussian, distinguished by heavy tails ${ }^{13}$. An approach to such distributions ${ }^{14}$ is the alpha-stable distributions, which generalize the gaussian distribution for the case of heavy tails. In these distributions, dispersion takes the place of variance as a measure of spread and minimum dispersion serves as the criterion of optimality in analogy with minimum variance in the gaussian case.

This document surveys the status of ambient ocean noise models and closes with recommendations based on the results of that survey. 


\section{SHALLOW WATER NOISE: MODEL BUILDING AND VALIDATION}

To be successful in developing useful models of ambient noise, the two processes of building (theory and simulation) and validation (comparison of model predictions with experimental data) both must be carried out. It may be said that the model building process is not completed until the validation succeeds broadly, that is, over a range of regional diversity likely to be encountered by the model user. Model based processing ${ }^{15}$ will be invaluable in dealing with imperfectly known regions but only if that region is an admitted member of the class encompassed by the model. The critical test of the model is that it prove consistent, in its formulation and assumptions, with the nature of the environment at hand: thus the necessity of obtaining good experimental data taken with model validation in mind.

This report deals primarily with the part of model building which is theory and simulation but the above is cited as a caution.

\section{A. Physical Models}

As mentioned in the introduction the modeling of shallow water noise has taken two forms, which we have termed as physical and stochastic.

Any treatment of the physics of noise must include the following aspects.

1) Source description.

2) Source aggregation.

3) Channel characterization.

4) Reception by the receiver

It is suggested that a physics-based noise model may be built by following these steps. Each step is described briefly below. 
Source description identifies a radiating source element whether it is a discrete source such a simple source ${ }^{16}$ in acoustics or a differential element of a continuous source ${ }^{17}$. It establishes the temporal variation of source strength which usually also establishes the frequency spectrum via a Fourier transform.

Source aggregation determines the spatial distribution and correlation properties of the sources identified above. Very general frameworks exist but , in practice, simple models ${ }^{18}$ assume a Poisson spatial and temporal distribution of uncorrelated sources.

Channel characterization involves the transmission channel which is comprised of the medium, its boundaries and its stratification, natural or imposed. An example of imposed stratification is the layering of oceans due to the assumption that sound velocity varies with depth in a piecewise linear dependence. While this assumption facilitates computation it leads to well-known artifacts (false caustics) in the solution for the acoustic field. Natural stratification can result in real caustics, as is well known.

The usual characterizing entity is the channel impulse response, which becomes the Green's function in the case of space $\left(\mathbf{r}^{\prime}\right)$ and time, $t^{\prime}$, discrete distribution of the form, $\delta \mathbf{r}^{\prime}$ $\delta t^{\prime}$ where the prime indicates that these quantities are connected with the source. Normally the receiving point pressure $p(r, t)$ is assumed to be related in time to the source pressure $p\left(r^{\prime}, t^{\prime}\right)$ by a time delay only, i.e, $\tau=\left(t-t^{\prime}\right)$. This results in the simple source with a pressure field given by $\mathrm{p}=1 / 4 \pi \int_{\mathrm{V}} \partial \mathrm{Q} / \partial \mathrm{t} \tau / \mathrm{r} \mathrm{dV}^{\prime}$, where $\mathrm{r}$ is a vector connecting the source and receiver point, $\mathrm{V}^{\prime}$ is the volume of the source and $\partial \mathrm{Q} / \partial \mathrm{t}$ is the mass flow rate in and out of $\mathrm{V}^{\prime}$.

Addition of nearby boundaries (less than a couple of wavelengths away) complicates the picture as now the interaction between the simple source and its image affects the radiation and makes invalid the simple source assumption. The simple source becomes a multipole source, a dipole in the case of perfect reflection at a free boundary. 
Reception by a point receiver is embodied in the Green's function solution to the linear wave equation with point excitation and with suitable boundary conditions. Reception by distributed receivers is the province of array theory: it has a rich literature in both acoustic and electromagnetic radiation fields. Most noise reception theories begin with the idea of a point receiver. If the array response is desired, the noise is estimated at each point receiver, assumed fixed or moving, and the resulting pressure at the array output is estimated through the response of aggregated receivers. ${ }^{19}$

In the example of the simple source above, analysis of reception by an array would require knowledge of radius vectors connecting all source and receiver points. Furthermore, in the inhomogeneous, refracting channel, (one with spatial variation in its properties) only the differential vector, $\mathrm{dr}$, is known so that the paths between source and receiver must be determined by an integral.

An example of model building and its consequences upon validation is given by Buckingham et. al. ${ }^{20}$ The steps followed are summarized:

1) Source description. Impulsive, fixed point sources with Poisson time occurrence of the impulses.

2) Source aggregation. All point, discrete sources lie in a plane, parallel to and very near a smooth sea surface plane and Poisson distributed spatially over that plane.

3) Channel characterization. A layered channel, of atmosphere (an acoustically free surface, a water column which is spatially homogeneous, a saturated fluid bottom and an elastic basement .

4) Reception by the receiver. Two hydrophones, with vertical separation of $1 \mathrm{~m}$ within the channel.

The theoretical analysis ${ }^{21}$ focuses on the coherence (normalized cross spectral density) of the noise at the two hydrophones and develops the solution in terms of radiating and attenuative normal modes. The surprise is, that in comparing the experimental data with the model, a better fit is achieved by ignoring the contribution of the attenuative, shortrange modes. Quoting, "This implies that little short range energy is present in the data, in contrast with the predictions of the model." At this writing the anomaly was unresolved. 
The lesson here is , "validate with data the assumptions in your model." Of special interest to the subject of sea surface interaction with acoustic or em waves is the follow-on analysis of the data to determine the actual occurrence rate of the impulses from capping and breaking waves. One question to be answered is, what is a sufficient observation time for the mean rate of impulses to be measurable? This is a live issue ${ }^{22}$ in the modeling of ambient noise in shallow water. Some time ago the question arose in deep water acoustics and was satisfied by the development of a white cap index ${ }^{23}$ which essentially is the fraction of area covered by capping waves. The following figure would represent an asymptotic value for shallow water waves as the water depth increases and thus as the effect of the sea bottom on the waves diminishes.

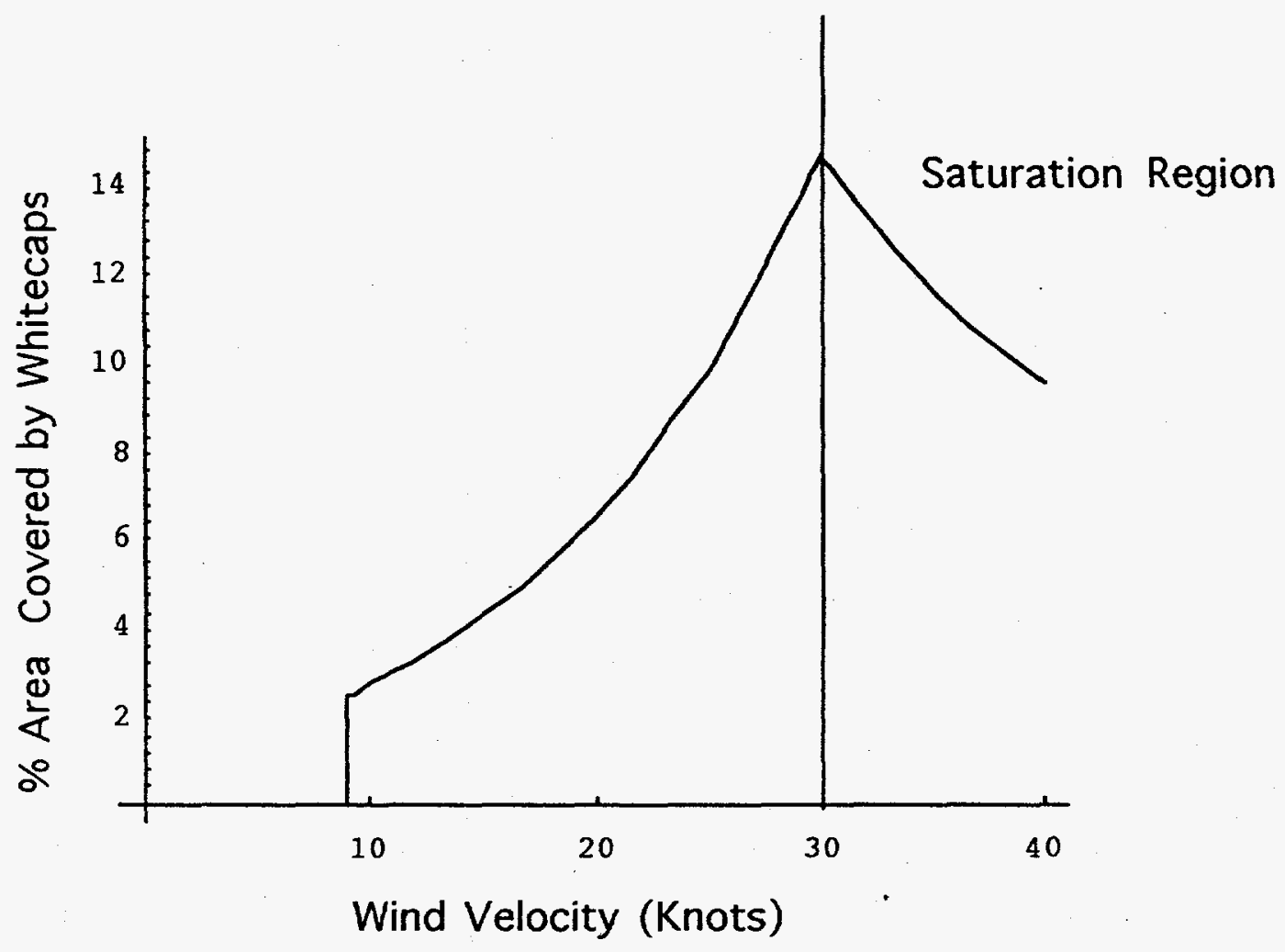

Noise source mechanisms arising from wave-wind interaction ${ }^{24}$ include ocean spray, streaks, whitecaps and rainfall in ways not fully understood but for which some theory and measurement exists. 


\section{B. Stochastic Models}

This category of model really is a part of all model building. It is concerned primarily with the determination of the probability distribution or density function of the noise considered as a random process. The random process is described ${ }^{25}$ by a hierarchy of density functions $\left\{W_{n}\right\}$, based on the noise process as an ensemble of noise waveforms, $\left\{y^{(n)}\right.$ t\}.

The following is a very brief introduction to this subject and follows Middleton. More discussions are available ${ }^{26}$.

\section{Distribution densities}

The first order probability density, $w_{1}\left(y_{1}, t_{1}\right)$ is determined first by letting the ensemble contain a finite number $M$ of waveforms, where $M$ is a large number. Then $y$ is divided into a set of $N<M$ units, each of small width, $\Delta y$. Then a level interval $\left(y_{1}, y_{1}+\Delta y\right)$, chosen at a time $t_{1}$ where $y_{1}=1 \Delta y$. We count the number of waveforms that attain this interval at this time, the number $n_{1}(M)$. The probability measure associated with this interval at this time is

$$
r_{1}=n_{1}(M) / M=P\left(y_{1}<y<y_{1}+\Delta y ; t_{1}\right)
$$

Then, taking limits $M->\infty, \Delta y->d y$, we assume that $n_{1}(M)->\infty$ in such a way that $r_{1} \rightarrow W_{1}\left(y_{1}\right.$, $\left.t_{1}\right) d y_{1}=$ probability that $y$ at time $t_{1}$ falls in the interval $\left(y_{1}, y_{1}+\Delta y\right)$.

Allowing $y_{1}$ to range over all possible values at time $t_{1}$, repeating the count for each value and the suitable limits, assumed to exist, gives $W_{1}\left(y_{1}, t_{1}\right)$.

\section{Marginal densities}

$\mathrm{W}_{1}$ is recognized as a first-order marginal density, since it involves only a fixed time. Higher order marginal densities are derived by a similar process to the one that produced that of first order, except that the intervals in $y$ are now joint and joint occurrences are 
counted within a set of $n$ intervals and $n$ times to yield $W_{n}\left(y_{1}, t_{1}, y_{2}, t_{2} ; \ldots ; y_{n}, t_{n}\right)$. These marginal densities provide a mathematical description of the random noise process. However it is impractical, given any real waveform set, to carry out this counting and limiting process to all orders.

\section{Conditional densities}

By a change of notation we express the idea of a conditional density. The simplest is, for second order,

$W_{2}\left(y_{1}, t_{1}, y_{2}, t_{2}\right)=$ probability that if $y$ at time $t_{1}$ falls in the interval $\left(y_{1}, y_{1}+\right)$ then $y$ at time $t_{2}$ will fall in the interval $\left(y_{2}, y_{2}+\Delta y\right)$.

This strikes one as more of a theorem than a definition. It is a statement of conditional sufficiency but not of necessity. The notation here (following Middleton) is opposite to the more common $W(x \mid y)$ that is read, probability of $x$ given $y$.

Marginal densities of higher order are related to those of lower order through the conditional densities. For instance,

$W_{2}\left(y_{1}, t_{1}, y_{2}, t_{2}\right)=W_{1}\left(y_{1}, t_{1}\right) W_{2}\left(y_{1}, t_{1}, \mid y_{2}, t_{2}\right)$

The Markoff process is one for which it may be written

$W_{n}\left(y_{1}, t_{1}, y_{2}, t_{2}, \ldots ; y_{n-1}, t_{n-1} l y_{n}, t_{n}\right)=W_{2}\left(y_{n-1}, t_{n-1} \mid y_{n}, t_{n}\right)$

As Middleton states it, "Markoff processes are important to noise model building since most noise processes are of this type."

\section{Mixed Processes}




\section{RECENT MODELS}

In this section some specific models of ambient ocean noise are summarized. A progression follows the shift in emphasis from deep to shallow water and advances in general computing capability.

\section{A. Cron And Sherman}

The Cron and Sherman model, ${ }^{29}$ while being a model for a deep water channel with no bottom, describes noise in terms of a distribution of point directional sources with a angular dependence that is an integer power of the cosine squared of the angle of radiation with the normal direction. Two-dimensional isotropic noise ${ }^{30}$ gives a normalized crossspectral density that is a Bessel function of zero order. $\operatorname{Cox}^{31}$ solves for the 3-dimensional noise field in terms of a directional density function that is a superposition of plane waves in all directions but not with equal strength and admitting noise from angles both positive and negative with respect to the horizontal. In a separate report, $\operatorname{Cox}^{32}$ argues for a composite model based on linear combinations of the cross-spectral density function and presents a number of them with some comparisons with data, all in deep water, but designed to give more realistic results than strictly models of analytic form. This is a caution that no one analytic model is likely to result in a practical model for use even in deep water, let alone shallow water.

\section{B. Kuperman \& Ingenito}

Kuperman \& Ingenito ${ }^{33}$ develop a model for calculation of the spatial properties of a noise field in stratified ocean. The source is a monopole of velocity potential. The source aggregation is an infinite plane of continuously distributed sources of uniform strength at a constant depth below a pressure relief surface. The channel is a three-layer acoustic medium of infinite horizontal extent, characterized by a sound velocity that depends upon depth but not range and a density fixed within each layer. The top layer is semi-infinite in height with zero density. The lowest layer is of infinite depth, with zero shear rigidity. It is claimed that more complicated channels can be accommodated by this model. In fact, later 
in the development, the necessity for attenuation is stated. Stratification is required to ensure separability of the wave equation.

It is assumed that both the source function and the wave function have Fourier transforms. Then these forms are substituted in a wave equation with random source function as a driver and a delta function in depth. The inhomogeneous Helmholtz equation is then solved for the frequency-dependent wave components in terms of the Fourier-transformed source function (source spectral strength) and a Green's function in range and depth of source and receiver. The cross spectral density is ensemble average of the magnitude squared of the wave spectral components. The transverse Fourier form of the Green's function is used to simplify the cross-spectral density of the noise field which then takes on a rather general form.

Then spatial stationarity of the source distribution is assumed. A noise spatial correlation function is introduced and transformed for convenience. The final result is a computable expression for the noise cross-spectral density. It has a simple form when the noise is delta correlated in the source-source horizontal separation.

To obtain a normal mode representation The Green's function is expanded in terms of normal modes and the modes with continuous wavenumber are assumed not to contribute. Thus the problem is to determine the discrete eigenvalues of a differential equation in the mode functions with boundary conditions. At this point the wavenumbers are assumed to be complex with the imaginary part being the attenuation coefficient. This is to avoid the paradox in a channel with cylindrical spreading loss and with a surface noise contribution that is growing with radius squared. The Green's function is inserted and the complex integration is carried out. It is argued that the crossterms in the residue series do not contribute to the sum since they represent mode-mode interference. Thus only the self terms are kept in the final formulation. This final form is strongly dependent on the value used for attenuation coefficient as well as the functional form of the spatial correlation of the noise field.

Several computed examples are given illustrating simple models of sound speed profiles in the central medium : isovelocity, linear downward refracting and piece-wise linear 
upward refracting. The sub-bottom is typical of sand-silt-clay mixture, $20 \mathrm{~m}$ thick overlying a rigid bottom. Appendix $B$ has a solved example of an isovelocity central medium and a rigid bottom.

\section{Buckingham}

Buckingham ${ }^{34}$ develops normal mode solutions of the range-independent velocity potential. He also assumes ${ }^{35}$ the "low-loss" channel in which the attenuation is finite but low enough that the distant sources predominate over the local sources. This produces a sound structure predominated by arrivals at low angle. Contributions from only discrete modes are retained whose "angles" may be given as a function of the bottom properties and the in-water wavenumber.

He also transforms the velocity potential and obtains the field in terms of a sum over modes. Then he specializes to receivers separated vertically and computes the differential cross-spectral density integrated for all ranges beyond a certain minimum which he immediately approximates to zero. After some simplification he obtains an expression for cross spectral density at two vertically separated receivers. His specific interest is in calculating the array gain of a vertical array of receivers from the cross spectral density. He shows explicitly that the cross-terms in the modal series may be neglected in agreement with Clay. He then obtains an approximation that does not depend specifically on the receiver depths only their separation, valid if the array is not too close to the boundaries. Upon normalization, the cross-spectral density no longer depends upon the attenuation and the final expression is one composed of measurable parameters, depth, speed of sound in the channel (isovelocity), and critical angle of the bottom.

By. imposing quasihomogeniety in the channel, a directional density function is developed using a relationship by Cox relating the cross spectral density and the direction density function. This function is a discrete angular distribution from which the noise gain of the array may be computed. 


\section{Jensen, Kuperman, Porter and Schmidt}

Jensen, Kuperman, Porter and Schmidt ${ }^{36}$ devote chapter 9 to the discussion of surface generated ambient noise, especially in shallow water, (ocean areas that are shoal enough for the occurrence of sensible interaction of the acoustic field with the local bottom and subottom.) This treatment omits discrete sources of sound such as commercial shipping and other marine activities with the comment that " the resulting acoustic field is just a straightforward, though involved, summation of acoustic fields from geographically distributed discrete sources."

Cross spectral methods and normal mode methods are first applied to the channel with range-independent characteristics which permit a discussion of the features of the noise: continuous spatial spectrum or mode number for the "overhead" noise; discrete modes for long-distance contributions; and evanescent wave contributions from seismo-acoustics. Next the channel with three-dimensional-dependent (3D) properties as well as a surface distribution with spatially varying source strength is treated by means of the adiabatic normal modes. Finally they discuss a parabolic equation approximation (PE) to the propagation applied to estimation of deep-ocean vertical noise directionality, ${ }^{37}$ valid for azimuthal symmetry. It is indicated that by reformulation the PE method might be applied also to the channel with $3 \mathrm{D}$ variation of spatial properties. This is apparently the present horizon of the state of the art.

\section{E. Kewley, Browning and Carey}

Kewley, Browning and $\mathrm{Carey}^{38}$ compare various measured and modeled data on the subjects of source characterization and aggregation. They treat only the source spectral pressure levels, with no regard for the fine points of phase relationships source distribution inhomogeneity or source correlation. They find some consistency in the data descriptions provided that a uniform method of describing source directionality is employed. Basic to the conceptual model of sound radiation from the sea surface is the notion $^{39}$ that the sound arises from a planar aggregation of non interacting, point 
monopoles held at some short distance below a pressure-release surface. Since in this picture the source dipole strength is derived by assuming a finite depth, it is necessary to determine that "effective depth". The authors select one quarter wavelength since this separation maximizes the vertical value of pressure from a true dipole ${ }^{40}$. The maximum is achieved for the condition $h=1 / 4+m, m=0,1,2 \ldots$

Why the minimum value was selected is not stated. The other question left untreated is the effect of the disturbed sea surface on the image source as the wind speed increases. Other analysis shows that this Lloyd mirror is modified by the Rayleigh parameter, $\mathrm{R}=\mathrm{kH}$ $\operatorname{Sin}(t), t$ is the angle of grazing incidence, $H$ is the rms wave height measured peak-totrough and $k$ is the wavevector magnitude. At normal incidence, $\operatorname{Sin}(t)=1$. For a fullydeveloped, deep sea ${ }^{41}$ it is shown theoretically that the effective reflection coefficient is given by $\mu=\exp (-\mathrm{R})$. For instance, at $600 \mathrm{~Hz}$ the acoustic wavelength is $1500 / 600=2.5 \mathrm{~m}$. $k=2 * 3.14 / 2.5=2.5, \mathrm{kH}=1$ at $\mathrm{H}=0.4 \mathrm{~m}$ or sea state $2(0.3 \mathrm{~m}-0.6 \mathrm{~m}), \mu=\exp [-\mathrm{R}]=.366$.

The effect of $\mu<1$ can be estimated from the Lloyd mirror reduced range $\mathrm{e}^{42}$ parameter $\rho=$ $r \lambda / 4 \pi d_{1} d_{2}$, where $d_{1}, d_{2}$ are the source and receiver depths and $r$ is the horizontal range. The equation for acoustic intensity ratio where $I_{o}$ is the intensity of the direct sound alone is

$$
I / I_{o}=1+\mu^{\wedge} 2-2 \mu \operatorname{Cos}[1 / \rho]
$$

The figure shows transmission loss in decibels as a function of $\rho$.

It is evident from the table in the figure that the dipole-forming image is degraded even at low wind speeds. 


\begin{tabular}{|l|c|c|}
\hline Curve & $\mu$ & ms Waveheight, $\mathrm{m}$ \\
\hline A & 0.1 & 0.9 \\
B & 0.5 & 0.27 \\
C & 1.0 & 0 \\
\hline
\end{tabular}

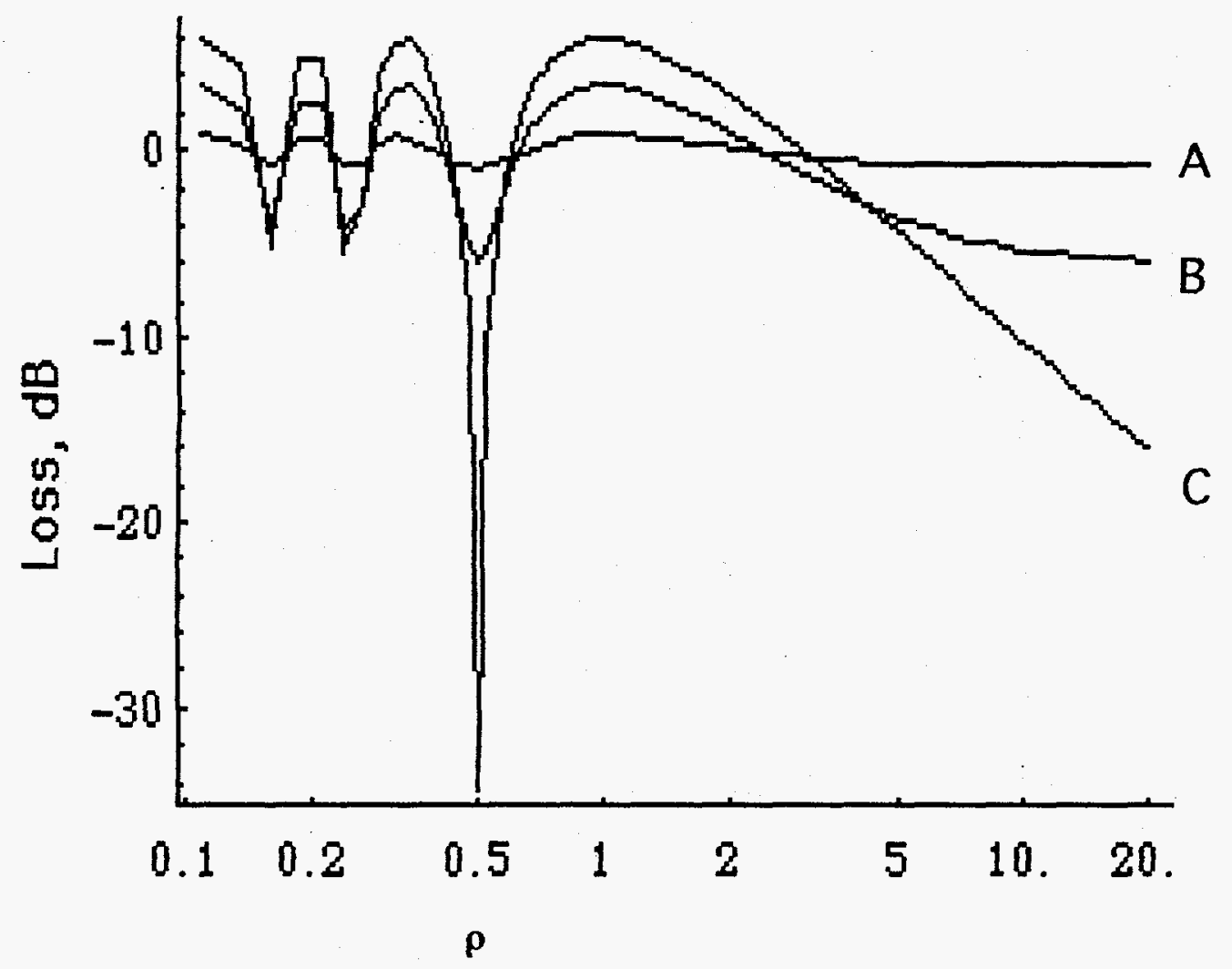

If the separation between source and image is held constant in wavelengths the dipole strength will also be constant and the dipole pattern will be the same.

In the far field, the intensity of dipole radiation shows inverse 4th power spreading loss with increasing range. However, at higher wind speeds, the surface reflection, being increasingly diffuse as wind speed increases, will weaken the dipole moment in favor of the monopole which has a inverse 2 nd power spreading in a free field. Cylindrical spreading is inverse 1st power of the horizontal range. Empirically, in shallow water, all of these spreading laws are used depending upon the range. At intermediate range, the inverse power of 1.5 is used for spreading $\operatorname{loss}^{43}$. In any case, the spreading loss for sea states greater than zero is progressively less that of a dipole and thus the contribution to the noise at long range should increase as the wind speed increases. At a sea state where 
the image is completely obscured a saturation effect from the contribution of the scattered sound would occur. This would result in an underestimation of the noise at higher wind speeds by any model that depends strictly upon dipoles as sources of sound.

\section{F. Piggot}

C. L. Piggot ${ }^{44}$ treated measured noise frequency spectra in shallow water (in 20 and 28 fathoms, Scotian Shelf, North Atlantic). He reports data from this year-long study including comparisons of spectral levels at two hydrophones (about $1.7 \mathrm{~nm}$ apart), the relationship between ambient sea noise and wind speed and the seasonal variation of the noise. Spectral curves are given which have been quoted widely as representative of open, shallow seas. The main results from Piggott indicate that:

1) Noise levels at the same frequency due to wind generally are higher in shallow water than in deep water (using Wenz ${ }^{45}$ as a reference for deep water values.) However in comparison with noise in much shallower water ${ }^{46}$ ( 7 fathoms, Narragansett Bay) the noise levels, compared at 25-knots wind speed, are lower below $400 \mathrm{~Hz}$ in the still more shallow water. These differences might be explained by the differences in propagation conditions where the shoal bottom is cutting off the higher order modes. This implicates long-range propagation in the establishment of local noise levels. Long range, in this instance, is of the order kilometers in shallow water. If equipartition is assumed, and if the total number of modes is inversely related to frequency then the difference between the two sets of data below a certain frequency should grow by $3 \mathrm{~dB} /$ octave of frequency decrease. This appear to be the case at 25 knots below $400 \mathrm{~Hz}$ (Piggott, Fig. 12).

2) The changing seasons affect average spectral level but not the slope of wind speed dependence. Shallow water noise for a given wind speed is $3.5 \mathrm{~dB}$ higher on the average at all frequencies during the winter. No detailed explanation was given for this difference, although it was speculated that the local sound speed profile was acting to lower the attenuation during the winter. This is because an upward refracting (winter) profile would favor surface interaction over bottom interaction with a resulting lower attenuation in 
the winter than in the summer. Surface heating, in the summer, acts to reverse the profile slope and produce more bottom interaction, thus higher attenuation.

These arguments again imply that long-range propagation-- the low-loss case of Kuperman and Ingenito-- more greatly influences the noise in the winter while, by the same argument, the short-range, "overhead" noise may dominate in the summer.

However, Kuperman and Ingenito ${ }^{47}$ indicate that frequencies around $500 \mathrm{~Hz}$ that the loss due to scattering from the disturbed sea surface dominates over that of a smooth bottom when upward refraction dominates and a reasonable wind is blowing. The $3.5 \mathrm{~dB}$ is not explained by this analysis.

Furthermore in one measurement study ${ }^{48}$ in shallow water $(30 \mathrm{~m}$, North Sea, $90 \mathrm{~m}$ Baltic Sea) the conclusion was reached that the propagation had only a marginal effect on noise levels and that the overhead noise dominated in most cases. The critical item appeared to be the surface wind velocity, controlled by the shape of the temperature gradient in the marine boundary layer. The wave height itself had little to do with the noise but was an indication of the changing wind profile. This is an appealing description because it is easy to imagine the shape of the marine boundary layer as a seasonal effect. Also it would call for a change in strategy in making shallow water noise measurements from concentration on sea water temperature profiles to measuring the sea air temperature in the marine boundary layer. The marine boundary layer is affected by the presence or absence of nearby land masses, a significant aspect of the littoral regions, which would strengthen the case for positing a local nature to ambient underwater noise.

"Over head" noise was separated from the distant noise contribution, however in deep water, ${ }^{49}$ (four sites, East of Australia), by beamforming a vertical array of hydrophones. The result was a separate look at the contributions to the source level of noise arriving from high and low angle with adjustment for local bottom loss. With low angle noise arrival excluded and bottom loss accounted for and at wind speeds up to 40 knots and in the frequency range of $100 \mathrm{~Hz}$ to $600 \mathrm{~Hz}$, flat spectral source levels were measured with a definite wind speed dependence given by $S=37.3+15 \log w, w$ the wind speed in knots, 
and $\mathrm{S}$ measured in $\mathrm{dB}$ re $1 \mu \mathrm{Pa}^{2}$ /steradian-Hz. Incidentally, below $100 \mathrm{~Hz}$ there is a significant source, not related to wind, which is also "over head"and unexplained. 


\section{MEASURED NOISE}

Noise in nature is an abstract concept. In practice, we deal with measured noise, i.e. that which has been transformed by a transducer and some electronic circuitry into a varying electrical current. Thus the noise of interest is really a transformation on the abstract noise ensemble. If the response of the measuring system is known, the input noise correlation, (a second-order statistic of the distribution) may be estimated from the system output and the system impulse response as is well known. But the input distribution itself may not be so easily estimated from the output. Middleton recognizes two classes of transformations: measure-preserving, in which the hierarchy of distribution densities is preserved, and non-measure-preserving, wherein these densities are altered by the system. The latter is the much more common case.

A principal problem is that any system has a transient as well as an equilibrium (steadystate) response so that the system output distribution may be changing with time and thus may not have a final form. To proceed with analysis of this sort the master equation ${ }^{50}$ is written in terms of the transition probability function $T\left(x, x^{\prime}\right) d x^{\prime} d t$, the probability that if the output has value $x$ at time $t$ then it will have value $x^{\prime}$ a short time $d t$ later. The transitional probability is a conditional probability. Then the master equation is written

$\int\left\{-f(x, t) T\left(x, x^{\prime}\right)+f\left[x^{\prime}, t^{\prime}\right) T\left(x^{\prime}, x\right)\right\} d x^{\prime}=\partial f[x, t] / \partial t$.

This equation is explained as follows. We which to assess the probability of a measurement state $x$, which by the counting method explained earlier would have us determine the fraction of waveforms in the ensemble that have this state at any observation time, $t$. The first term accounts for all waveforms in the ensemble which leave the state $x$ for the state $x^{\prime}$ in the time interval $\mathrm{dt}$, a loss of those waveforms in the counting process. The second term accounts for those waveforms that arrive at state $x$, from state $x^{\prime}$, a gain in the counting process, and the integral sums over all the values of $x^{\prime}$. 
The method is applied to a transducer measuring thermal noise in the ocean The master equation when applied to thermally driven processes such as Brownian motion, simplifies to a Fockker-Planck form

$\lambda B \partial(f x) / \partial x+k T B \partial^{2} f x / \partial x^{2}=\partial f / \partial t$

where $\lambda$ is the restoring force of the transducer to a force, F, assumed to arise from the acoustic pressure, $\mathrm{p}$, and $\mathrm{B}$ is the mobility of the water particles, driven by the noise pressure field and impinging on the face of the hydrophone. If no elastic force is applied, i.e., the transducer is absent, then $\lambda=0$ and the particles merely diffuse with diffusion constant, kTB. For the steady-state distribution $\partial f / \partial t=0$ the Fokker-Planck equation. becomes (independent of the mobility of the particle)

$$
\lambda \partial(f x) / \partial x+k T \partial^{2} f x / \partial x^{2}=0
$$

with gaussian solution

$$
f(x)=(\lambda / 2 \pi k T)^{1 / 2} e^{-\lambda \times 2 / 2 k T}
$$

The approach to equilibrium is studied as follows. A sub-ensemble of noise waveforms is selected such that the initial value is a constant, $x=x_{0}$ over all the chosen waveforms. Then the solution to the Fokker-Planck equation, is

$$
f\left(x, x_{0}, t\right)=\left(\lambda /\left(2 \pi k T ( 1 - \operatorname { e x p } ( - 2 \lambda B t ) ) ^ { 1 / 2 } \operatorname { e x p } \left[-\lambda\left(\left(x-x_{0}\right) \exp (-\lambda B t)^{2}\right) /(2 \pi k T(1-\exp (-2 \lambda B t))] .\right.\right.\right.
$$

This distribution is highly peaked about $x_{0}$ for $t<<\lambda B$ and approaches a gaussian for $t>>1$ / $\lambda B$.

Since good noise measurement practice requires long observation times, the conclusion would be that the distribution is gaussian, missing the fluctuation dynamics going on. For instance, the (time-dependent) mean and mean square are

$$
<x>=\int x f d x=x_{0} \exp (-\lambda B t),
$$




$$
<x^{2}>=\int x^{2} \mathrm{fdx}=\mathrm{kT} / \lambda+\left(\mathrm{x}_{0}{ }^{2}-\mathrm{kT} / \lambda\right) \exp (-2 \lambda \mathrm{Bt}) .
$$

For $t<<\lambda B,<x^{2}>=2 k T B t$, the Einstein equation of displacement under Brownian motion. The importance of thermal noise at high frequencies has been long recognized and was treated for the acoustic case by Mellin ${ }^{51}$. He obtains a frequency spectrum

$$
<\mathrm{P}^{2}>\mathrm{df}=4 \pi \mathrm{f}^{2} / \mathrm{c}^{2} \mathrm{kT} \rho \mathrm{cdf}
$$

where, here, $\mathrm{f}$ is the frequency. This results in a noise level ${ }^{52}$ of

$$
-15+20 \log _{10} \mathrm{f}-\mathrm{DI}-\mathrm{E} \text { expressed in } \mathrm{dB} \text { re } 1 \mu \mathrm{pa}
$$

for a hydrophone of directional gain, DI, and efficiency, $\mathrm{E}$, of converting acoustic energy to electrical energy. This equivalent noise exceeds the quietest ambient sea noise at about $20 \mathrm{kHz}$ and above.

The above is a rich source of distribution densities that stem from the general approach to their determination as follows, paraphrasing Middleton $^{53}$, of obtaining d.d's from a physically fundamental viewpoint by studying the (stochastic) differential equations which govern the random process.

The approach taken here forks on whether the system, excited randomly, is linear (differential equation approach preferred) or nonlinear (postulational approach preferred). Middleton's Table 10.1, p460, displays d.d.s for four different physical models of the random walk problem for particle with various force fields. The case of the harmonically bound particle would be most appropriate to the linear transducer immersed in the acoustic field, etc. 


\section{PRACTICAL NOISE MODELS}

For applications in sonar performance the Navy (SPAWARS) relies on the SPARS model, developed over the years and in many versions by the Applied Research Laboratory, University of Texas. Among the many contributors to this model which is designed to operate in the field, are David Grant (noise measurement and studies, Carole Shepard (fast normal mode methods) and Evan Westwood (range-dependent PE propagation). Beyond these models, the ANDES (Ambient Noise Directionality Estimation System) developed at SAIC ${ }^{54}$ and the RANDII (Random Ambient Noise Directionality) model, developed at NRaD, NATO Saclant Center and NRL, Stennis Space Center and associated principally with $R$. Wagstaff, form the group of modern ambient noise models that are recognized if not endorsed by the US Navy community. 


\section{FINDINGS}

Based on this survey the following conclusions are stated:

1) There is no standard ambient noise model for shallow water. Ambient noise models in use compute averaged intensity, as a function of the arrival direction of the sound.

2) There is a class of model in use, whose members differ in their details but all follow the scheme. (a) Source description, (b) Source aggregation, (c) Channel characterization, (d) Reception by the receiver. The favored source is a point source which produces a dipole when combined with its surface image. The aggregation for surface noise is a continuous, uniform sheet of dipoles. The data base for source levels and distributions for shipping noise is known as HITS, developed for deep water and for which there is no shallow water counterpart. The preferred channel characterization is through the Green's function or impulsive response yielding discrete normal modes. A line hydrophone array is the most usual receiver.

3) The sound propagation model most in use is the Normal Mode model wherein the continuous modes are ignored. As an alternative the Parabolic Equation (PE) approximation is employed in several versions that attempt to account for radiation at high grazing angles.

4) There is no generic shallow water region for practical model computation, however, useful information concerning noise character may derived from some simplifying assumptions. Local environmental characteristics should be considered so that any simulations may relate to the particular region in mind. 


\section{RECOMMENDATIONS}

It is recommended that Lawrence Livermore National Laboratory perform the following analysis in order to derive the best understanding of noise for application to underwater signal processing.

1) Obtain data on ambient noise in shallow water for which the most complete characterization of the area has been made in terms of the sources of sound, their spatial distribution, the local character of the channel and the characteristics of the receiver.

2) Fit a probability distribution to these data, based on some chosen scheme such as alphastable distributions.

3) Chose a channel description for some region of shallow seas.

4) Chose a model of sound propagation.

5) Develop a computer simulation that produces noise pressure at any receiving point within the channel. The Buckingham model is a good staring point.

6) Characterize the probability distribution from the simulation time-series.

7) Compare the measured distribution with that of the simulation to fix parameter values and ranges .

8) Utilize the resulting simulation in model-based processing to determine the performance degradation due to the degree of nongaussian behavior of shallow water noise.

A further recommendation is that research be conducted on the form of optimal filtering of nongaussian noise using, in particular, the minimum dispersion criterion for alphastable nongaussian noise for application to radar and sonar.

${ }^{1}$ R. C. Cavanaugh and W. W. Renner, "Vertical Directionality and depth dependence of averaged acoustic signals and noise", J. Acoust. Soc. Am., 68 (5), 1467-1474, November 1980 ${ }^{2}$ A specific activity is at ARL:UT, under the direction of Clark Penrod.

${ }^{3}$ Person in charge is Ed Chiaka, currently not supporting noise modeling work at ONR.

${ }^{4}$ Program descriptions are published from time to time at ONR.

${ }^{5}$ Dick Heitmeyer is leading this effort at NRL under the program title, "Ambient Noise in full spectrum processing." 
${ }^{6}$ Analysis of this data is continuing at NRL in FY 1996.

${ }^{7}$ P. M. Woodard, Probability Theory with Applications to Radar, p24-25, (Pergamon Press, New York, 1953)

${ }^{8}$ D. Gabor, "Theory of communications," J. Inst. Elec. Engrs, 93 III, 429,(1946), 94, III 369

(1947) makes the further connection between noise fluctuations and distributions clear.

${ }^{9}$ D. Middleton, Introduction to Statistical Theory of Communications, Peninsula Publishing, Los Altos, CA (1987)

${ }^{10}$ Active systems in shallow water are usually considered to be reverberation limited. Reverberation, which results from scattering in the channel and from its boundaries, might be considered as signal-dependent noise in a generalized framework where background noise becomes the signal-independent component. Interference with the search signal, by signal-like returns from inhomogeneities present in the channel, then are included as discrete components of the reverberation. see Middleton's latest book for a thorough discourse on this subject,

${ }^{11}$ I. Deyer, "Statistics of distant shipping noise," J. Acoust. Soc. Am., 53 (5), 564-570, 1973 ${ }^{12}$ M. Buckingham, G. B. Deanne And. N. M. Carbone, “Determination of elastic sea floor parameters from shallow-water ambient noise, 2nd European Conference on Underwater Acoustics, L. Bjonro Ed.

${ }^{13}$ W. Michael and C. S. Penrod, probability density functions of ocean noise processes, in Statistical Signal processing, Ed. Wegman, Ed. 211-221 M. Dekker, 1984. See also, W. Machell, C. S. Penrod and G. E. Ellis, "Statistical characteristics of ocean noise processes," in Topics in non-gaussian signal processing, E. J. Wegman, et. al. ed. 29-57, Springer, New York, 1989

${ }^{14}$ C. L. Nikitas and Min Shao, Signal Processing with alpha-stable distributions and applications, John Wiley and Sons, to appear.

${ }^{15} \mathrm{~J}$. V. Candy and E. J. Sullivan, “Ocean acoustic signal processing: a model-based Approach," J. Acoust. Soc. Am. 92(6) 3185-3201 December 1992 ${ }^{16}$ Kinser and Frey, Fundamentals of Acoustics, 2nd Ed., 163, John Wiley and Sons, New York (1962)

${ }^{17}$ W. Kuperman and F. Ingenito, "Spatial Correlation of surface generated noise in a stratified ocean, Acoust. Soc. Am., 67 (6), 1987-1996 (June 1980) 
${ }^{18}$ Buckingham, loc. cit.

${ }^{19}$ Normally this involves beamforming. There are approaches that aggregate the receivers without beamforming as such. See E. Sullivan.

${ }^{20}$ M. J. Buckingham, G. B. Deanne And. N. M. Carbone, "Determination of elastic sea floor parameters from shallow-water ambient noise, 19-25, 2nd European Conference on Underwater Acoustics, L. Bjonro, Ed.

${ }^{21} \mathrm{M}$. J. Buckingham, "A theoretical model of ambient noise in a low-loss, shallow water channel," J. Acoust. Soc. Am.,67, 1186-1192 (1980)

${ }^{22}$ David M. Farmer and Li Ding, "Recent developments in the passive detection of breaking surface waves," 27-32, 2nd European Conference on Underwater Acoustics, L. Bjonro, Ed. (1994)

${ }^{23}$ J. H. Wilson, "Wind-generated noise modeling," J. Acoust. Soc. Am., 73, 539-545 (1983. Wilson gives a formula (validated by data) by which this index may be estimated for deep water. The threshold wind speed is 9 knots below which no whitecaps appear. The next range, determined empirically, is given by the wind velocity $U$ for $9 k t<U<30 k t$ as $R[U]=$ $U^{3} / 1749.60-U^{2} / 81.0+U / 4.32^{\star} 1.5$, and a saturation range for $U>30 \mathrm{kt}$ as $(R(30) / U / 30)^{1.5}$.

${ }^{24} \mathrm{~J}$. H. Wilson, "Low frequency wind-generated noise produced by the impact of spray with the ocean's surface," J. Acoust. Soc. Am., 68(3) ,952-956 (September 1980)

${ }^{25}$ D. Middleton, Introduction to Statistical Theory of Communications, Peninsula Publishing, Los Altos, CA (1987)

${ }^{26}$ Middleton, op. cit.

${ }^{27}$ Middleton, op. cit. $\mathrm{p} 7,8$

${ }^{28}$ F. Borghini, G. Tacconi and A. Tiano," A comparative H.O.S. estimation of Class B nongaussian underwater acoustic noise. 45-50, 2nd European Conference on Underwater Acoustics, L. Bjonro, Ed. Mention is made of a Matlab toolbox for the processing of nonlinear and nongaussian signals. See G. Tacconi , A. Tiano and F. Borghini," A Matlab toolbox for the processing of non-linear and non-gaussian signals," I.A.N. (CNR-Genoa), Genoa University, 1992.

${ }^{29}$ B . F. Cron and C. H. Sherman, J. Acoust. Soc. Am. 34, 1732-1736, (1962), 38 885(L) (1965)

${ }^{30}$ M. Jacobson, J. Acoust. Soc. Am. 34, 971-978 (1962)

${ }^{31}{ }^{31} \mathrm{H}$. Cox, "Spatial correlation in arbitrary noise fields with application to ambient sea noise," J. Acoust. Am. 54(5), 1289-1301, (1973) 
${ }^{32} \mathrm{H}$. Cox, "Ambient noise models," Underwater Sound labs report no number.

${ }^{33}$ W. A. Kuperman and F. Ingenito, "Spatial correlation of surface generated noise in a stratified ocean," J. Acoust. Soc. Am. 67 (June 1980) 1988-1996

${ }^{34}$ M. J. Buckingham," A theoretical model of ambient noise in a low-loss shallow water channel, J. Acoust. Soc. Am. 67(4) APRIL 1980 1186-1192

35 as does Kuperman and Ingenito

${ }^{36}$ F. Jensen, W. Kuperman, M. Porter, H. Schmidt, Computational Ocean Acoustics, ATP Press 1994

${ }^{37}$ W. M. Carey, R. B. Evans, J. A. Davis and G. Botseas, “Deep-ocean vertical noise directionality," IEEE J. Ocean Eng. OE-15 , 324-334 (1990)

J Kewley, D. G. Browning, and W. M. Carey, "Low frequency wind-generated ambient noise source levels, J. Acoust. Am. 88 (4), 1894-1902, October 1990

${ }^{39}$ B. F. Cron and C. H. Sherman, op. cit.

${ }^{40}$ As the bandwidth increases the plane becomes a layer under this assumption

41 For a shallow sea the significant wave height, itself, is a function of the water depth in the region of the observation. See: M. L. Khandekar, Operational Analysis and Prediction of Ocean Wind Waves, 104 et. seq., Coastal and Estuarine Studies 33, Springer-Verlag 1989. ${ }^{42}$ R. J. Urick, Principles of Underwater sound, p124, McGraw Hill 1975 ${ }^{43}$ H. W. Marsh and M. Schulkin, "Shallow-water transmission," J. Acoust. Soc. 34 (6), 863864, June 1962

${ }^{44}$ C. L. Piggott , Ambient sea noise at low frequencies in the shallow water of the Scotian Shelf," J. Acoust. Am. 36 (11) 2152-2163 (November 1964)

${ }^{45}$ G. M. Wenz, J. Acoust. Soc. Am. 34 ,1936-1956 (1962)

${ }^{46}$ F. T. Dietz, J. S. Kahn and W. B. Birch, J. Acoust. Soc. Am. 32, 915 (A) (1960

${ }^{47} \mathrm{~W}$. Kuperman and F. Ingenito, "Attenuation of coherent sound in shallow water," J. Acoust. Soc. Am. 81 (5) ,1178-1187 May 1977

${ }^{48}$ P. C. Wille and D. Geyer,"Measurements on the origin of the wind-dependent ambient noise variability in shallow water," J. Acoust. Soc. Am. 75(1) ,173-185 (January 1984). ${ }^{49}$ A. S. Burgess and D. J. Kewley, "Wind-generated surface noise levels in deep water east of Australia," J. Acoust. Soc. Am. 73(1) ,201-210 (January 1983).

${ }^{50}$ Mac. Donald, Noise and Fluctuations : an Introduction „, p 69 et seq., John Wiley \& Sons ¡New York (1962) 
${ }^{51}$ Robert H. Mellin, "The thermal noise limit in the detection of underwater acoustic signals," J. Acoust. Soc. Am., 24, 478-480 (1952)

${ }^{52}$ R. J. Urick, Principles of underwater sound, p 187, (McGraw Hill, New York, 1975)

${ }^{53}$ Middleton, chapter 10, p 438, et. seq.

${ }^{54}$ W. W. Renner, "Ambient Noise Directionality Estimation System (ANDES), Technical Description," SAIC Technical Report No. SAIC-86/1645, Science Applications Corporation, McLean VA (June 1986) 